\title{
SIRCM1, which encodes tomato Lutescent1, is required for chlorophyll synthesis and chloroplast development in fruits
}

\author{
Genzhong Liu', Huiyang Yu', Lei Yuan', Changxing Li', Jie Ye®l', Weifang Chen'1, Ying Wang ${ }^{1}$, Pingfei Ge',
} Junhong Zhang ${ }^{1}$, Zhibiao Ye(i] ${ }^{1}$ and Yuyang Zhang (10

\begin{abstract}
In plants, chloroplasts are the sites at which photosynthesis occurs, and an increased abundance of chloroplasts increases the nutritional quality of plants and the resultant color of fruits. However, the molecular mechanisms underlying chlorophyll synthesis and chloroplast development in tomato fruits remain unknown. In this study, we isolated a chlorophyll-deficient mutant, reduced chlorophyll mutant 1 ( $\mathrm{rcm} 1)$, by ethylmethanesulfonate mutagenesis; this mutant produced yellowish fruits with altered chloroplast development. MutMap revealed that Solyc08g005010 is the causal gene underlying the $\mathrm{rcm} 1$ mutant phenotype. A single-nucleotide base substitution in the second exon of SIRCM1 results in premature termination of its translated protein. SIRCM1 encodes a chloroplast-targeted metalloendopeptidase that is orthologous to the BCM1 protein of Arabidopsis and the stay-green G protein of soybean (Glycine max L. Merr.). Notably, the yellowish phenotype of the lutescent1 mutant can be restored with the allele of SIRCM1 from wild-type tomato. In contrast, knockout of SIRCM1 by the CRISPR/Cas9 system in Alisa Craig yielded yellowish fruits at the mature green stage, as was the case for lutescent1. Amino acid sequence alignment and functional complementation assays showed that SIRCM1 is indeed Lutescent1. These findings provide new insights into the regulation of chloroplast development in tomato fruits.
\end{abstract}

\section{Introduction}

Fruit development is a complex and highly coordinated process that involves a series of specific physiological and biochemical changes ${ }^{1,2}$. During tomato fruit development, chloroplasts serve as sites of photosynthesis and carbohydrate accumulation and can be transformed into chromoplasts for carotenoid formation ${ }^{3}$. Chloroplast development in tomato plants is directly proportional to fruit development and nutrient accumulation. Both the synthesis and degradation of chloroplasts in plants are in a stable state of dynamic equilibrium. Therefore, it is of enormous importance to study the molecular mechanism underlying chloroplast development in the process of fruit ripening and development.

\footnotetext{
Correspondence: Yuyang Zhang (yyzhang@mail.hzau.edu.cn)

${ }^{1}$ Key Laboratory of Horticultural Plant Biology, Ministry of Education, Huazhong Agricultural University, Wuhan, China
}

Chloroplast development has been demonstrated to be regulated by multiple transcription factors, among which GLK2 is an influential transcription factor that regulates this process. Tomato fruits with full-length transcripts of GLK2 mRNA exhibit a dark green shoulder that can promote photosynthesis and accumulate more nutrients, while $g l k 2$ mutation eliminates this green shoulder ${ }^{4}$. GLK2 overexpression resulted in dramatic upregulated expression of the Solyc08g005010 gene, indicating a regulatory network involving chloroplast development ${ }^{5}$. The TKN2 and TKN4 genes in tomato modulate the gradient of chloroplast development in fruits by regulating GLK2 expression $^{6}$. Overexpression of the APRR2-like gene in tomato increased the content of chlorophyll in fruits ${ }^{7}$. $S I B B X 20$ in tomato regulates chloroplast development by modulating the expression of the SlCAB1B,SlCAB6A and $S l C H L 27$ genes $^{8}$. SlBEL11 encodes a transcription factor

\section{(c) The Author(s) 2021}

(c) (i) Open Access This article is licensed under a Creative Commons Attribution 4.0 International License, which permits use, sharing, adaptation, distribution and reproduction cc) in any medium or format, as long as you give appropriate credit to the original author(s) and the source, provide a link to the Creative Commons license, and indicate if changes were made. The images or other third party material in this article are included in the article's Creative Commons license, unless indicated otherwise in a credit line to the material. If material is not included in the article's Creative Commons license and your intended use is not permitted by statutory regulation or exceeds the permitted use, you will need to obtain permission directly from the copyright holder. To view a copy of this license, visit http://creativecommons.org/licenses/by/4.0/. 
that negatively regulates chloroplast development and chlorophyll synthesis in tomato fruits. The SlBEL11 protein can directly bind to the promoters of genes involved in chloroplast development and chlorophyll synthesis, such as TKN2 ,CAB and POR, and downregulate the expression of these types of genes ${ }^{9}$. In plant growth and development, auxin signaling affects chlorophyll synthesis and chloroplast development. The auxin response factor ARF10 directly binds to the promoter of GLK1 and activates its expression, thereby promoting chloroplast development and sugar accumulation ${ }^{10}$. SIARF6A directly binds to the promoters of the GLK1,CAB1,CAB2 and $R b c S$ genes and promotes their expression to modulate tomato fruit chloroplast development ${ }^{11}$. ARF2A, an auxin signal component, participates in fruit ripening and chloroplast development. In Arabidopsis, the ARF2 gene is involved in the degradation of chlorophyll in leaves ${ }^{12}$. The chlorophyll content of ARF2A-OX transgenic fruit was significantly lower than that of wild-type fruit at 42 days post-anthesis ${ }^{13}$.

Chlorophyll synthesis in plants is a complex process involving 15 enzymes encoded by 27 genes $^{14}$. Mgchelatase catalyzes the binding of $\mathrm{Mg}^{2+}$ to protoporphyrin IX, which represents the first step in chlorophyll synthesis ${ }^{15}$, and Mg-protoporphyrin IX is further methylated by Mg-protoporphyrin IX methyltransferase, followed by four subsequent catalytic reactions to produce chlorophyll ${ }^{16}$. Chlorophyllide a oxygenase (CAO) catalyzes the conversion of chlorophyll $a$ to chlorophyll $b$ and plays an important role in the balance of chlorophyll $a$ and chlorophyll $b$. Overexpression of $C A O$ in tobacco promotes the synthesis of chlorophyll $b$ and decreases the ratio of chlorophyll $a$ and chlorophyll $b^{17}$. GUN4, a porphyrin-binding protein, enhances the activity of $\mathrm{Mg}$ chelatase by binding to protoporphyrin, which is the substrate of the Mg-chelatase reaction ${ }^{18}$. Overexpression of GUN4 can significantly increase the chlorophyll content in tobacco leaves ${ }^{19}$. SGR1, encoding Mg dechelatase, promotes chloroplast degradation during plant maturation $^{20}$. Mutation of SGR1 in Chinese cabbage leads to increased chlorophyll concentrations and a stay-green phenotype $^{21}$. In Arabidopsis, the $b c m 1$ mutant exhibits a pale-green leaf phenotype due to reduced chlorophyll contents. BCM1 interacts with GUN4 to enhance Mgchelatase activity. BCM1 can also interact with SGR1 to destabilize the SGR1 protein $^{22}$. GluTR is a glutamyl tRNA reductase involved in porphyrin and chlorophyll biosynthesis $^{23}$. Interactions between BCM1 and GluTR affect the synthesis of 5-aminolevulinic acid, a key precursor in the biosynthesis of porphyrin during chlorophyll synthesis $^{22}$. The product of CHLM is magnesium protoporphyrin IX methyltransferase that converts $\mathrm{Mg}$ protoporphyrin IX to Mg-protoporphyrin IX methylester during chlorophyll synthesis ${ }^{24}$. BCM1 interacts with
CHLM to promote the formation of a MgCH-GUN4CHLM enzyme complex ${ }^{22}$. The stay-green $G$ gene is a homologous gene of $B C M 1$ in soybean; this gene positively regulates chlorophyll synthesis in the soybean seed $\mathrm{coat}^{25}$. Therefore, BCM1 plays an important and conserved role in chlorophyll synthesis in different crop species.

In tomato, the Lutescent2 (L2) gene, which encodes a zinc metalloprotease, has been characterized, and this gene has been shown to regulate chloroplast development and fruit maturity ${ }^{26}$. Similarly, the number of chloroplasts per cell in immature green fruit pericarp tissue of lutescent1 (l1) mutant was reduced significantly compared with that of the wild type. In addition, fully expanded leaflets of the $l 1$ mutant exhibited a more dramatic yellowish phenotype ${ }^{26}$. It was previously reported that the chlorophyll content of the fruits of the $l 1$ mutant was reduced, leading to a senescent phenotype for $l 1^{27}$. Chloroplast development is hindered in $l 1^{28}$, although the causative gene underlying chloroplast defects and chlorophyll reduction in $l 1$ has not yet been identified.

In this study, we obtained a reduced chlorophyll mutant (rcm1) of tomato; this mutant is an $l 1$ allelic mutant with altered chloroplast development and was generated via ethylmethanesulfonate (EMS) mutagenesis. We discovered via BSA + DNA-Seq and MutMap that the $r \mathrm{~cm} 1$ mutant carries a single-nucleotide polymorphism $(\mathrm{T} \rightarrow$ A), resulting in premature termination of the SIRCM1 protein. Sequence analysis and functional characterization showed that the SIRCM1 gene, located at the Lutescent1 locus, encodes an ortholog of the BCM1 protein of Arabidopsis and the stay-green G protein of soybean (Glycine $\max$ L. Merr.). SlRCM1 regulates chlorophyll synthesis and chloroplast development in fruits at the mature green and red ripe stages, while its ortholog regulates chlorophyll synthesis in the leaves of Arabidopsis and seeds of soybean $^{22,25}$. These findings highlight the molecular mechanisms underlying chlorophyll synthesis and chloroplast development in tomato fruits.

\section{Results \\ The tomato $\mathrm{rcm} 1$ mutant exhibits altered chloroplast development}

Seeds of the tomato cultivar Ligeer 87-5 were treated with $1 \%$ EMS to obtain novel mutants. A reduced chlorophyll mutant $(\mathrm{rcm} 1)$ was isolated from the EMSmutagenized population (Fig. 1a). The fruits of the $\mathrm{rcm} 1$ mutant exhibited reduced amounts of chlorophyll relative to those of the wild type. The fruits at the mature green (MG) and breaker (BR) stages of the $r \mathrm{~cm} 1$ mutant showed a sharp decrease in chlorophyll relative to those of the wild type. However, the fruits of the $r c m 1$ mutant could still turn red at the red ripe (RR) stage (Fig. 1b). Furthermore, the thylakoid membranes were collapsed in the 
(a)

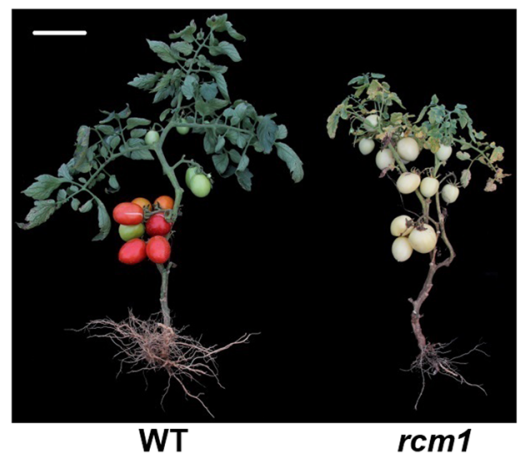

(c)

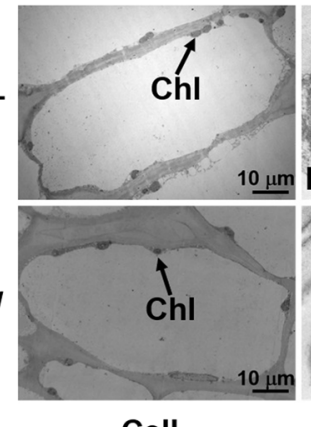

Cell

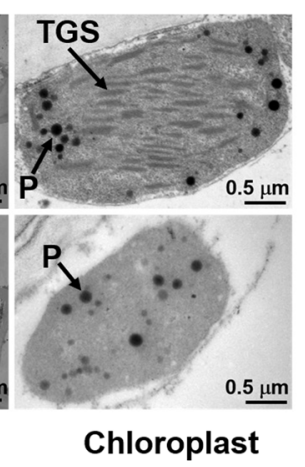

Chloroplast (b)

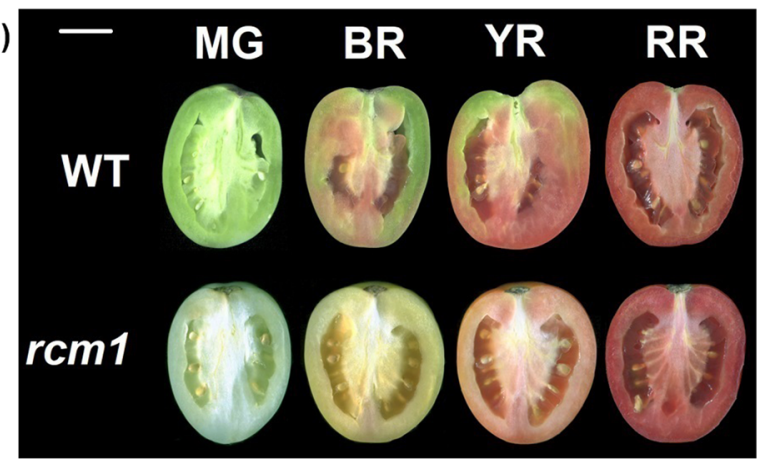

(d)

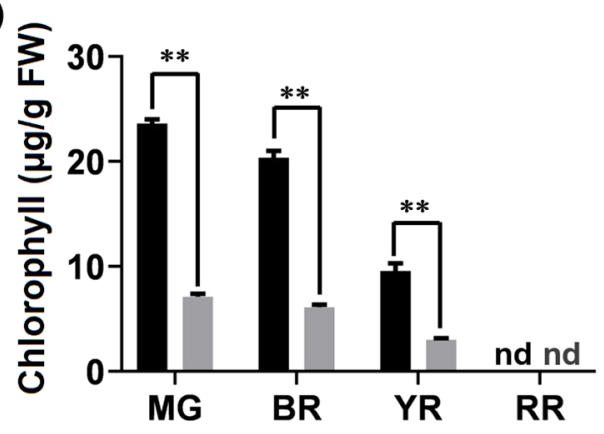

(f)

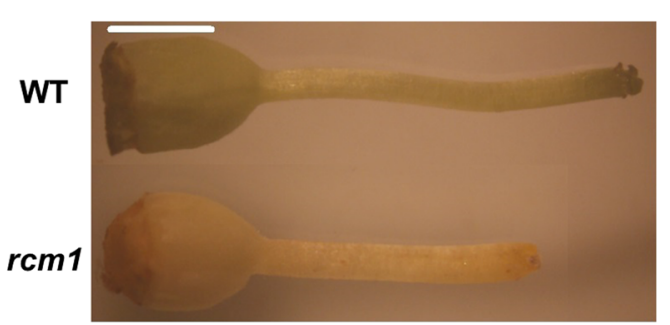

(i)

WT

rcm1

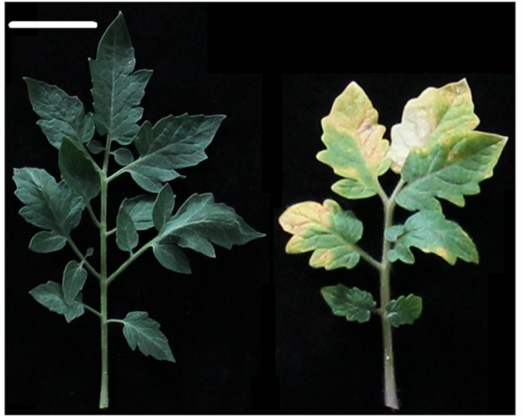

WT

rcm1 (h)

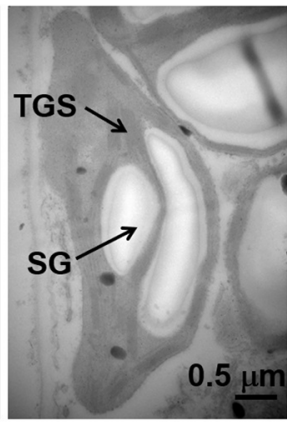

WT

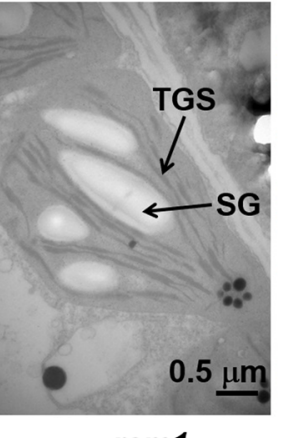

rcm1

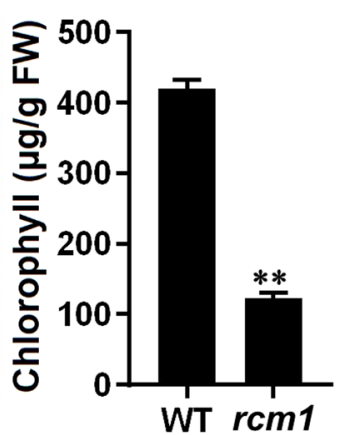

Fig. 1 Phenotypes of wild-type and $\mathbf{r c m} 1$ mutant plants. a Whole-plant morphology of the reduced-chlorophyll mutant $(\mathrm{rcm} 1)$ and the wild type (Ligeer 87-5). Scale bars, $15 \mathrm{~cm}$. b Phenotypes of Wild Type (WT) and rcm 1 tomato fruits at four developmental stages. MG, mature green stage; BR, breaker stage; YR, yellow ripe stage; RR, red ripe stage. Scale bars, $2 \mathrm{~cm}$. c Chloroplast ultrastructure of mature green fruits of wild-type and rcm 1 mutant plants via transmission electron microscopy. P, plastoglobuli; TGS, thylakoid granum stacks. Scale bars, $10 \mu \mathrm{m}$ (left) and $0.5 \mu \mathrm{m}$ (right). d Chlorophyll content of fruit pericarps from wild type and $r \mathrm{~cm} 1$ at the MG, BR, YR and RR stages. The data are presented as the means \pm SDs ( $n=6$ ). e Carotenoid contents of fruit pericarps from wild type and $r \mathrm{~cm} 1$ at the MG, BR, YR and RR stages. The data are presented as the means \pm SDs $(n=6)$. $\mathbf{f}$ Phenotypes of the pistils of the wild type and $\mathrm{rcm} 1$ mutant. Scale bars, $2 \mathrm{~mm}$. $\mathbf{g}$ Morphology of the leaves of the $\mathrm{rcm} 1$ mutant and the wild type. Scale bars, $2 \mathrm{~cm}$. h Chloroplast ultrastructure of leaves of wild-type and $\mathrm{rcm} 1$ plants using transmission electron microscopy. SG, starch grains; TGS, thylakoid granum stacks. Scale bars, $0.5 \mu \mathrm{m}$. i Chlorophyll content of leaves from wild-type and $\mathrm{rcm} 1$ plants. The data are presented as the means \pm SDs $(n=6)$. The asterisks indicate statistically significant differences according to $t$-tests: ${ }^{* *}, P$-value $<0.01$. nd, not detected 
chloroplasts of fruits of the $r \mathrm{~cm} 1$ mutant at the mature green stage (Fig. 1c). The chloroplasts in the $\mathrm{rcm} 1$ fruits were smaller than those of the wild type (Fig. 1c). Subsequently, the chlorophyll content in the $r \mathrm{~cm} 1$ mutant fruits was significantly lower than that in the wild-type fruits at the MG, BR and yellow red (YR) stages, and no chlorophyll was detected at the RR stage (Fig. 1d). The carotenoid content in the $\mathrm{rcm} 1$ mutant fruits was also significantly lower than that in the wild-type fruits at all four developmental stages (Fig. 1e). The $\mathrm{a}^{*} / \mathrm{b}^{*}$ colorimetric values (Fig. S1a), which are proportional to the lycopene, and total soluble solids contents (Fig. S1b) of red ripe fruits of the $r \mathrm{rm} 1$ mutant decreased relative to those of the wild type. Fruit ripening of the $r \mathrm{~cm} 1$ mutant was delayed by approximately 6 days compared to that of the wild-type (Fig. S2a), and the ethylene production rate of the $r \mathrm{rm} 1$ mutant fruits was lower than that of the wildtype fruits (Fig. S2b).

To fully evaluate the phenotype of the $r \mathrm{rm} 1$ mutant, the leaves and pistils were compared between the $\mathrm{rcm} 1$ mutant and wild type. A lack of chlorophyll accumulation was observed in the developing pistils and leaves of $\mathrm{rcm} 1$ relative to the wild type (Fig. 1f, g). Transmission electron microscopy revealed impaired development of thylakoid membranes in the chloroplasts of the $\mathrm{rcm} 1$ mutant compared to those of the wild type (Fig. 1h). Accordingly, the chlorophyll content in the $r \mathrm{~cm} 1$ mutant leaves was significantly lower than that in the wild-type leaves (Fig. 1i). Compared to that of the wild type, the maximum photochemical efficiency $(\mathrm{Fv} / \mathrm{Fm})$ of leaves of the $\mathrm{rcm} 1$ mutant decreased significantly (Fig. S3a, b). Additionally, the quantum efficiency of PSII (Y(II)) of the leaves of the rcm1 mutant was significantly impaired compared with that of the wild type (Fig. S3c). Additionally, pollen vitality in $r c m 1$ was lower than that in Ligeer 87-5 (WT); the percentage of malformed pollen in $\mathrm{rcm} 1$ was greater than that in the wild type (Fig. S4).

\section{Cloning of SIRCM1}

To genetically characterize the yellowish fruit phenotype of the $r c m 1$ mutant, we generated an $\mathrm{F}_{2}$ population consisting of 307 individuals by crossing Ligeer $87-5$ with the rcm1 mutant. The $\mathrm{F}_{1}$ generation of the cross between Ligeer 87-5 and $r c m 1$ displayed a normal phenotype similar to that of Ligeer 87-5, which suggested that the gene underlying the reduced chlorophyll phenotype of the $\mathrm{rcm} 1$ mutant is a recessive gene. In the $F_{2}$ population, the ratio between the number of individuals with a normal phenotype (237) and the number of individuals with a chlorophyll-deficient phenotype (70) was approximately $3: 1\left(x^{2}=0.68, x_{0.05}^{2}=\right.$ 3.84 ), indicating that the chlorophyll-deficient phenotype was controlled by a single gene (Table S1).

Next, we performed BSA and MutMap analyses to isolate the candidate genes. We sequenced two bulk populations comprising 25 individuals with green fruit or yellowish fruit at the mature green stage. Each library was sequenced at a depth of a $\times 25$ genome equivalent. The generated reads were mapped to the tomato reference genome (M82), and allele frequency differences of 40,124 SNPs from the two pools were calculated and mapped across the 12 chromosomes in tomato to form a Manhattan plot (Fig. 2c). The confidence threshold exceeded $95 \%$ only at the beginning of chromosome 8, between SL2.50ch08_1 and SL2.50ch08_1010000 (Table S2). Generally, single-base mutations are frequently generated by EMS mutagenesis ${ }^{29,30}$. There were six SNPs between the two pools between SL2.50ch08_1 and SL2.50ch08_1010000 (Table S3). Genetic analysis of the segregating populations indicated that the phenotype of the rcm1 mutant is likely controlled by a single locus. Since M82 (the genotype of the reference genome) develops normal chloroplasts in its fruits, SNPs in the recessive pool with an allele frequency of 1 and different from the reference genome (M82) were scored, and only one SNP $(\mathrm{A} \rightarrow \mathrm{T})$ at SL2.50ch08_16268 out of all SNPs in the coding regions was identified.

Furthermore, no InDels or other structural variations were detected via 25 equivalent genome sequencing events of the WT and $\mathrm{rcm} 1$ mutants. We analyzed the allele frequency of the SNP at SL2.50ch08_16268 in both pools with wild-type and mutant phenotypes. The SNP at SL2.50ch08_16268 in M82 was A, whereas in the mutant pool, it was $\mathrm{T}(100 \%)$ (Table 1). Furthermore, the SNP at SL2.50ch08_16268 in 30 $\mathrm{F}_{2}$ progeny with yellowish fruit was $\mathrm{T}(100 \%)$, which was confirmed via PCR and Sanger sequencing. This SNP (SL2.50ch08_16268) occurs in the second exon of Solyc08g005010 and was further verified by Sanger sequencing. Because the transcriptional direction of Solyc08g005010 is opposite to that of the genome, the nucleotide of SL2.50ch08_16268 in the coding strand of Solyc08g005010 in M82 and Ligeer 87-5 was T, but it was A in the $r c m 1$ mutant (Fig. 2d, e). The substitution of $\mathrm{T}$ (wild type) to A $(\mathrm{rcm} 1$ mutant) resulted in the conversion of Tyr (wild type) to a stop codon ( $\mathrm{rcm} 1 \mathrm{mutant}$ ) (Fig. 2e), indicating that the translation of the SIRCM1 protein was terminated prematurely in the $\mathrm{r} \mathrm{cm} 1$ mutant. The predicted protein encoded by the SIRCM1 allele from the $r c m 1$ mutant did not include any functional domain (Fig. S5a). Therefore, we inferred that the SIRCM1 allele in the $r \mathrm{rm} 1$ mutant is null. Furthermore, the expression level of the SIRCM1 allele in the $r \mathrm{rm} 1$ mutant was equivalent to that in the wild type (Fig. S5b). Therefore, we considered Solyc08g005010 to be the candidate SlRCM1 gene, which encodes a CAAX-type endopeptidase.

\section{The yellowish phenotype of fruits of $r \mathrm{rm} 1$ was complemented by SIRCM1 from Ligeer 87-5}

To verify whether SIRCM1 is the causal gene for the chlorophyll-deficient phenotype of the $\mathrm{rcm} 1$ mutant, SlRCM1 was overexpressed (OE) under the control of the 


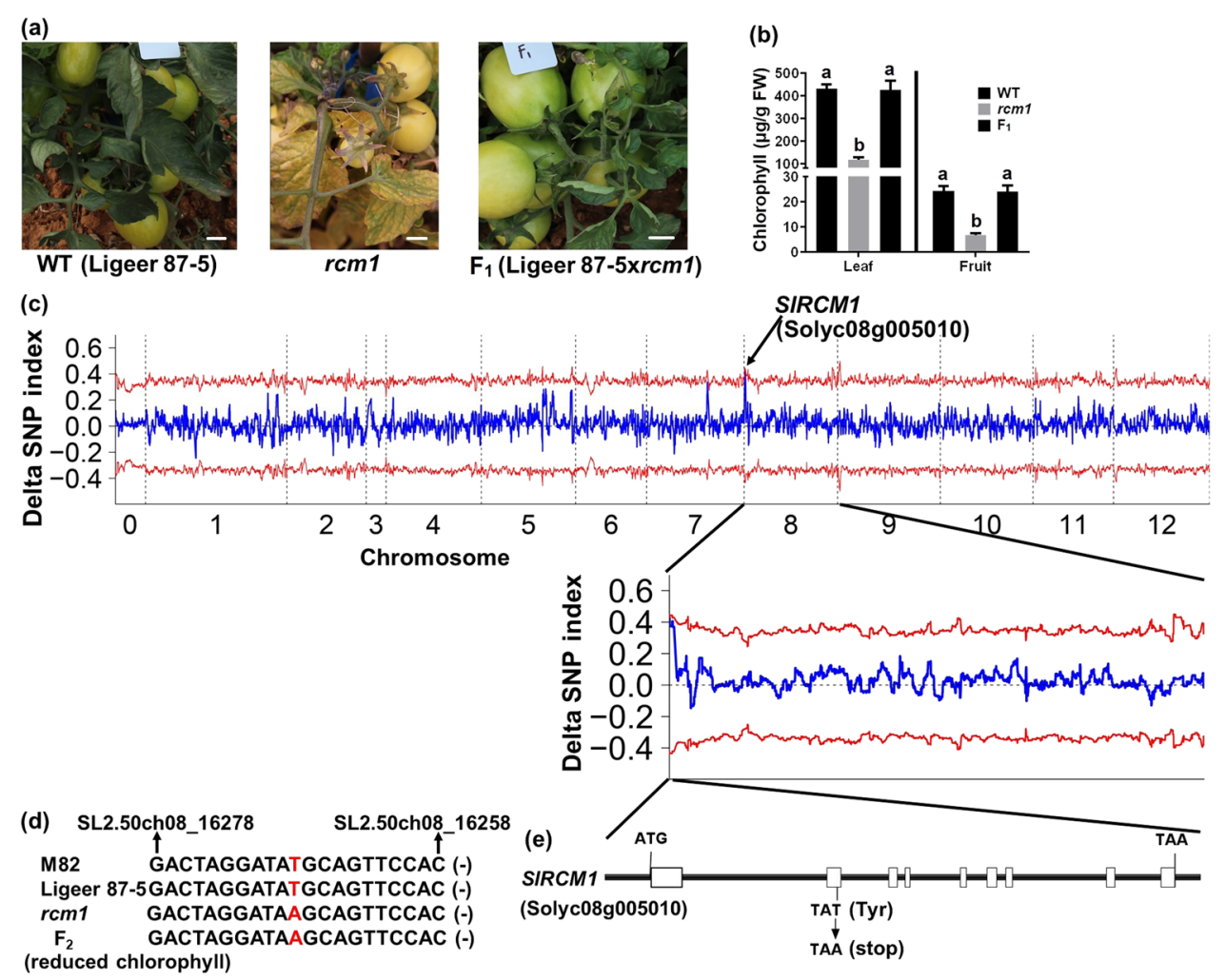

Fig. 2 Gene cloning, sequence variation and structure of the SIRCM1 gene. a Phenotypes of leaves and fruits from the wild-type and rcm 1 mutant plants and their $F_{1}$ progeny. Scale bars, $2 \mathrm{~cm}$. b Chlorophyll content of mature leaves and MG-stage fruits from the wild type and rcm 1 mutant and their $F_{1}$ progeny. The data are presented as the means \pm SDs $(n=3)$. The means followed by different letters indicate statistical significance at $P=0.05$. c Differences in allele frequencies between 25 lines with green fruits and 25 lines with yellowish fruits in the $F_{2}$ population. The $X$ axis represents the 12 chromosomes of tomato. The $Y$ axis represents the difference in allele frequencies (SNP values) between the two pools. The red line represents the 95\% confidence interval. The SNPs associated with the 95\% confidence interval are located between SL2.50ch08_1 and SL2.50ch08_1010000. The blue line represents the average value of the $\triangle S N P$ index per $1 \mathrm{Mb}$ window. $\mathbf{d}$ The fragment containing SNP (SL2.50ch08_16268) was subjected to PCR amplification and Sanger sequencing. The red letters indicate the bases of M82 (T), Ligeer 87-5 (T), rcm 1 (A) and members of the hybrid $F_{2}$ generation (A) with reduced chlorophyll at SL2.50ch08_16268. "-" indicates the antisense strand of the genome. e Structure of the SIRCM1 gene. The black lines represent introns and untranslated regions. The white boxes represent exons. The T (WT)-to-A (rcm 1) substitution in the second exon of SIRCM1 caused a conversion from Tyr (WT) to a stop codon ( $\mathrm{rcm} 1)$

Table 1 List of candidate SNPs and genes between SL2.50ch08_1 and SL2.50ch08_1010000

\begin{tabular}{llllllllll}
\hline Chr & Pos & Ref & Allele & $\begin{array}{l}\text { Wild } \\
\text { type index }\end{array}$ & Mutant index & $\begin{array}{l}\text { Delta } \\
\text { SNP index }\end{array}$ & Location & Gene & $\begin{array}{c}\text { Direction } \\
\text { Amino } \\
\text { acid change }\end{array}$ \\
\hline SL2.50ch08 & 16268 & A & $T$ & 0.27 & 1 & 0.73 & Exon & Solyc08g005010 Reverse & Tyr $\rightarrow$ Stop \\
SL2.50ch08 & 394612 & G & A & 0.42 & 1 & 0.58 & Intergene -- & -- & -- \\
\hline
\end{tabular}

CaMV35S promoter in the rcm1 mutant (Fig. 3a). Nineteen independent transgenic lines were obtained. The expression levels of the SlRCM1 gene in the OE-11 (\#11) and $\mathrm{OE}-18$ (\#18) lines in the $\mathrm{T}_{1}$ generation significantly increased relative to those of the $r \mathrm{~cm} 1$ mutant. The normal-green phenotypes of the fruits of the OE-11 and $\mathrm{OE}-18$ lines were restored at the mature green stage (Fig. $3 \mathrm{~b})$. To further verify the biological function of SIRCM1, a transformation construct (Pro SlRCM1 (Ligeer 87-5):: $\mathrm{CDS}^{\text {SlRCM1 (Ligeer }}{ }^{87-5)}$ ) was prepared by inserting the SIRCM1 CDS from Ligeer 87-5 into a pHELLSGATE8 vector under the native promoter of the transgene (Fig. 3c). We introduced this construct into the $\mathrm{rcm} 1$ mutant by Agrobacterium-mediated transformation. Eleven independent transgenic plants were identified. The phenotype of the $r \mathrm{~cm} 1$ transgenic lines was restored to that of the 


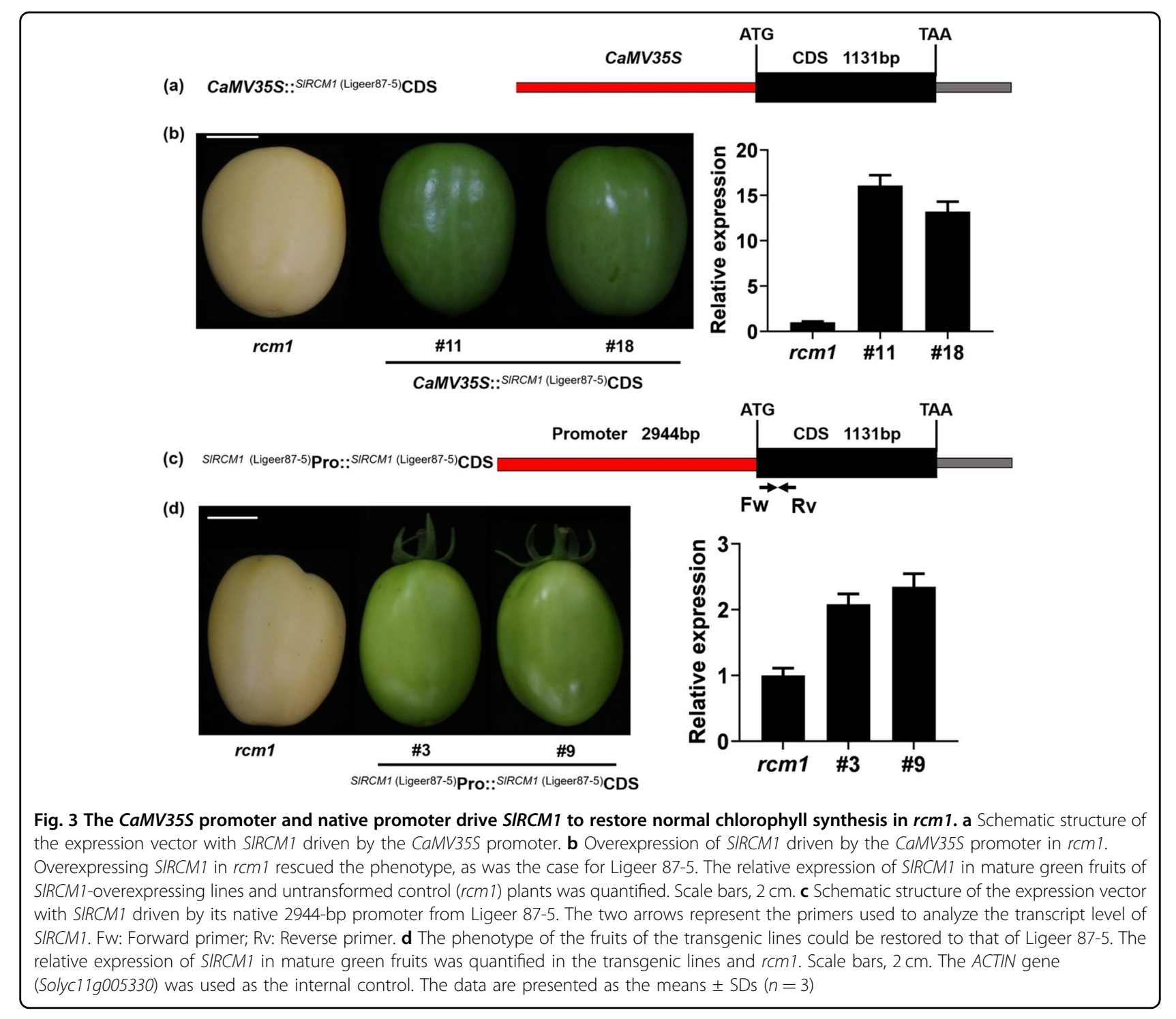

wild type (normal green fruit) (Fig. 3d). Overall, these results confirmed that SIRCM1 is the correct candidate gene.

\section{SIRCM1 regulates chloroplast development in tomato}

To further verify that SIRCM1 regulates chloroplast development in tomato fruits, we overexpressed the SIRCM1 gene in S. lycopersicum (L.) cv. Alisa Craig (AC) by Agrobacterium-mediated transformation. Compared to those of the wild type, the fruits of the OE-1 and OE-2 lines appeared dark green at the mature green stage (Fig. 4a). The number of thylakoids and thylakoid granum stacks in mature green fruits of the OE lines was higher than that of the wild type (Fig. 4b). Interestingly, the locular material surrounding the seeds remained green, and chlorophyll could still be detected at the red ripe stage in the overexpression lines (Fig. 4a). Indeed, the expression levels of the SIRCM1 gene in the OE-1 and OE-2 lines were significantly higher than those in the wild-type (AC) line (Fig. 4c). The chlorophyll content in the MG- and RR-stage fruits of OE-1 and OE-2 lines increased significantly relative to that in the wild type (Fig. $4 \mathrm{e}, \mathrm{f})$. In addition, the carotenoid content in the MG- and RR-stage fruits of the OE-1 and OE-2 lines increased significantly relative to that of the wild type (Fig. 4g, h). Chlorophyll in the RR-stage fruit of AC was not detected. Furthermore, we used CRISPR/Cas9 (CR) to edit the first exon of SlRCM1 in the AC background (Fig. 4a, d). We determined the type of mutation in the $\mathrm{T}_{1}$ generation using PCR and Sanger sequencing. The SIRCM1 knockout line CR-1 contained a 2-bp deletion in SIRCM1, and the knockout line CR-2 contained a 1-bp insertion in SIRCM1 (Fig. 4d). Both the CR-1 and CR-2 lines exhibited a reduced chlorophyll phenotype at and after the mature 
(a)

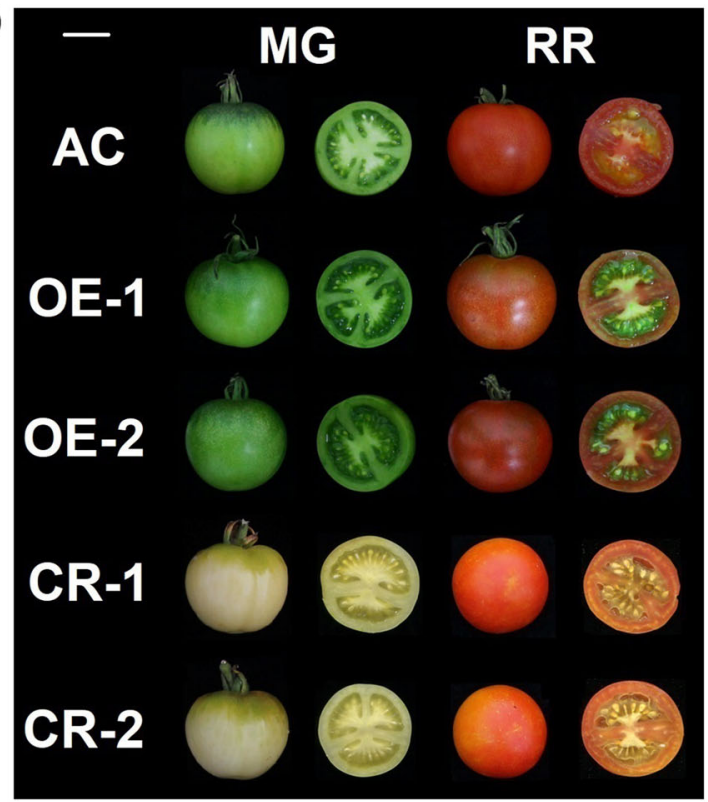

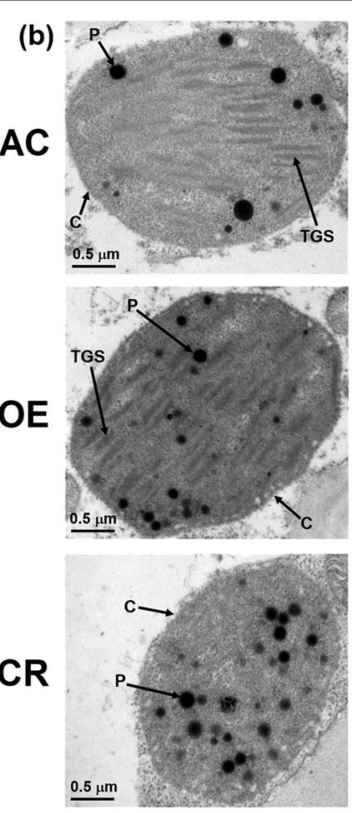

Target

PAM

(d) AC (SIRCM1) TTTCCGATTAGAAACAATTATCGAGA-TAAGAGG AGGAATTATA CR-1 (CR-SIRCM1-1) TTTCCGATTAGAAACAATTATCGA---TAAGAGG IGGAATTATA CR-2 (CR-SIRCM1-2) TTTCCGATTAGAAACAATTATCGAGATTAAGAGGAGGAATTATA

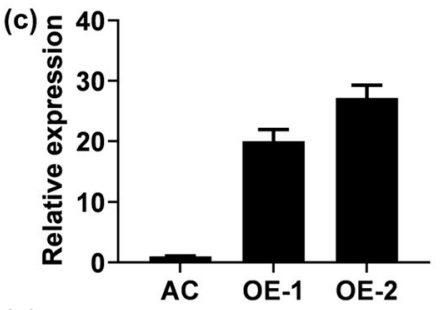

(e)

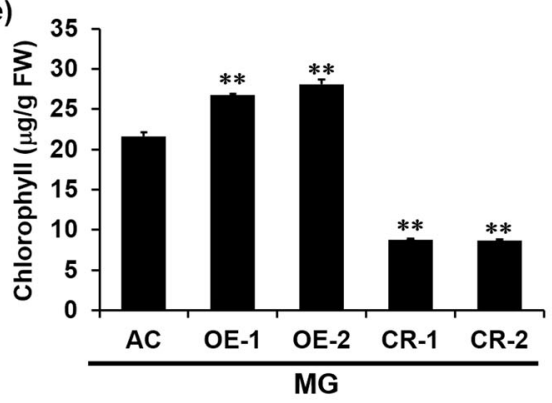

(g)

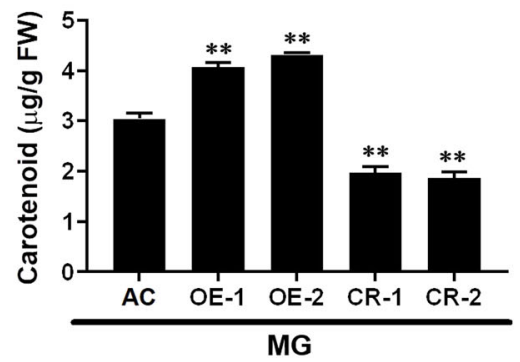

(f)

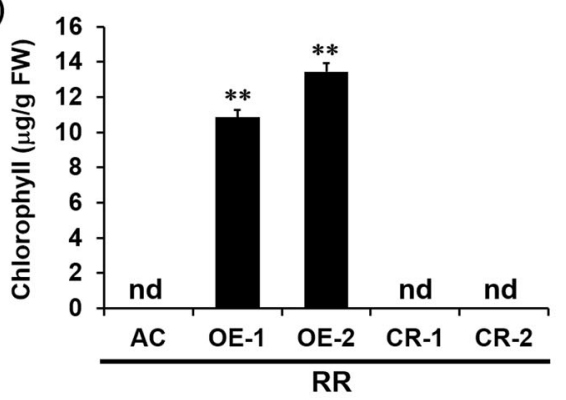

(h)

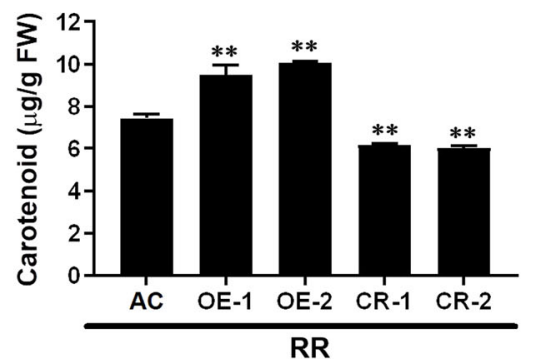

Fig. 4 Phenotypes of SIRCM1 transgenic lines and wild-type plants. a Fruits of AC, SIRCM1 overexpression and knockout lines at the mature green (MG) and red ripe (RR) stages. Scale bars, $3 \mathrm{~cm}$. b The structures of chloroplasts in mature green fruits of AC, SIRCM1-OE (OE) and SIRCM1-CRISPR (CR) were observed via transmission electron microscopy. C, chloroplast; P, plastoglobulus; TGS, thylakoid granum stack. Scale bars, $0.5 \mu m$. c The relative expression of SIRCM1 in mature green fruits of SIRCM1 overexpression lines and the control (AC). The ACTIN gene (Solyc11g005330) was used as the internal control. The data are presented as the means \pm SDs $(n=3)$. $\mathbf{d}$ Mutation types of SIRCM1 knockout lines in the $T_{1}$ generation were identified. e Chlorophyll content in the fruit pericarps of AC, SIRCM1-OE (OE) and SIRCM1-CRISPR (CR) lines at the mature green (MG) stage. $\mathbf{f}$

Chlorophyll content in the fruit pericarps of AC, SIRCM1-OE (OE) and SIRCM1-CRISPR (CR) lines at the red ripe (RR) stage. g Carotenoid content in the fruit pericarps of AC, SIRCM1-OE (OE) and SIRCM1-CRISPR (CR) lines at the MG stage. $\mathbf{h}$ Carotenoid content in the fruit pericarps of AC, SIRCM1-OE (OE) and SIRCM1-CRISPR (CR) lines at the RR stage. The data are presented as the means \pm SDs $(n=6)$. The asterisks indicate statistically significant 
green stage (Fig. 4a). Furthermore, the development of thylakoid membranes was impaired in the mature green fruits of the CR lines compared to that of the wild type (Fig. 4b). The chlorophyll content in the MG-stage fruits of CR-1 and CR-2 plants decreased significantly relative to that in the wild-type plants (Fig. 4e). These results indicate that SIRCM1 is responsible for chlorophyll synthesis and chloroplast development in tomato fruits.

\section{SIRCM1 is Lutescent 1}

A spontaneous lutescent1 mutant (l1), LA3717, was derived from Ailsa Craig (AC). The $l 1$ mutation has been shown to dramatically affect tomato fruit development. $l 1$ mutants have a low chlorophyll content in their fruits, especially under high-light and dark conditions, which enhances the rate of chlorophyll loss ${ }^{26}$. To date, the gene underlying the $l 1$ mutant has not yet been cloned. The fruits of SIRCM1 knockout lines (CR) generated using the CRISPR/Cas9 system exhibited the same yellowish phenotype as did the fruits of the $l 1$ mutant in the Alisa Craig (AC) background described in the Tomato Genetics Resource Center (TGRC; https://tgrc.ucdavis.edu) (Fig. 5a). The yellowish phenotype of the fruits of the $l 1$ mutant is purportedly controlled by a single gene located at the beginning of chromosome $8^{26,31}$. We used SIRCM1-specific primers to amplify full-length gDNA and the 5 -kb promoter of SIRCM1 from AC, Ligeer 87-5 and $l 1$ (Table S4). A base deletion led to premature termination of the SIRCM1 protein in $l 1$ (Fig. 5b, c). Furthermore, the expression level of the SIRCM1 allele in the $l 1$ mutant was equivalent to that in AC (Fig. S5c). SIRCM1 was subsequently overexpressed under the control of the CaMV35S promoter in the $l 1$ mutant. Nine independent transgenic lines were identified. The expression level of the SIRCM1 gene in the $2^{\text {nd }}(\# 2)$ and $6^{\text {th }}(\# 6)$ transgenic lines was significantly higher than that in the control (l1) (Fig. 5d). Functional complementation of SlRCM1 in $l 1$ restored normal plant growth and development (Fig. 5e). Taken together, these results indicate that SIRCM1 may be a causal gene underlying the yellowish phenotype of the $l 1$ mutant.

\section{SIRCM1 expression and subcellular localization}

The gDNA of the SlRCM1 gene is 6,299 bp in length and consists of nine exons and eight introns. The SIRCM1 gene encodes a protein comprising 376 amino acids. Alignment of amino acid sequences (Fig. S6) and phylogenetic analysis (Fig. 6a) showed that the SIRCM1 protein is highly conserved among soybean, potato, pepper, tobacco, Arabidopsis, rice, maize, jute and Cephalotus follicularis, suggesting that the SIRCM1 protein may play an essential role in plants. Tomato SIRCM1 may function in chloroplast development and chlorophyll synthesis through a conserved biological process, similar to that in soybean and Arabidopsis thaliana.
To investigate the expression pattern of SlRCM1, quantitative reverse transcription polymerase chain reaction (qRT-PCR) was performed as described previously ${ }^{32}$. SlRCM1 expression was detected in all tissues, and relatively high expression levels were detected in the leaves, flowers and immature green fruits (Fig. 6b). Furthermore, we used GUS staining to study the expression of SIRCM1 in several tissues. The GUS staining revealed that SIRCM1 was expressed in the stems, leaves, flowers and fruits (Fig. 6c).

To determine the SIRCM1 subcellular localization, we constructed a SIRCM1::GFP fusion protein. Through Agrobacterium-mediated infiltration, the SIRCM1::GFP fusion protein was transiently expressed in tobacco. Under a Nikon e600 fluorescence microscope (Nikon, Tokyo, Japan), the autofluorescence of the chloroplast appeared $\operatorname{red}^{33}$. The SIRCM1::GFP fluorescence overlapped with the red fluorescence, indicating that the SIRCM1 protein is localized in the chloroplast (Fig. 6d).

\section{The expression level of SIRCM1 is directly regulated by the transcription factor SIARF2A}

It was previously reported that $A R F$ proteins regulate gene expression by binding to the TGTCTC cis-element of the target gene promoter ${ }^{34}$. Yeast one-hybrid (Y1H) and dual luciferase experiments indicated that SIARF2A binds to cis-elements of the SIRCM1 promoter. The TGTCTC cis-element is located -245 to -239 bp upstream of the start codon of SIRCM1 (Fig. 7a). Yeast cells that were cotransformed with $p G A D T 7-S I A R F 2 A$ and $p A b A i$ SlRCM1-Pro were able to grow on $20 \mathrm{ng} / \mathrm{mL}$ aureobasidin A (ABA) SD/-Leu media, but the negative control cotransformed with $p$ GADT7 and $p A b A i$-SlRCM1-Pro did not grow (Fig. $7 \mathrm{~b}$ ), indicating that the ARF2A protein could directly bind to the promoter of SIRCM1.

To further indicate that the SIARF2A protein interacted with the promoter of SIRCM1 in plants, we constructed a luciferase (LUC) reporter harboring a promoter sequence from $-1,478$ bp to the start codon (ATG) derived from SIRCM1 (Fig. 7c). Tobacco leaves were coinfiltrated with Agrobacterium tumefaciens (GV3101) strains containing the indicated effector constructs containing SIARF2A and the reporter constructs containing the SIRCM1 promoter (Fig. 7d). Taken together, these results indicate that SIARF2A physically binds to the SIRCM1 promoter to downregulate its expression.

\section{Discussion}

Chlorophyll is essential for plants to absorb, transfer and convert light energy to bioenergy and plays a vital role in photosynthesis to promote plant growth and development ${ }^{35}$. Here, we cloned the SlRCM1 gene, which controls chlorophyll synthesis and chloroplast development in tomato fruits. A single-nucleotide replacement resulting 

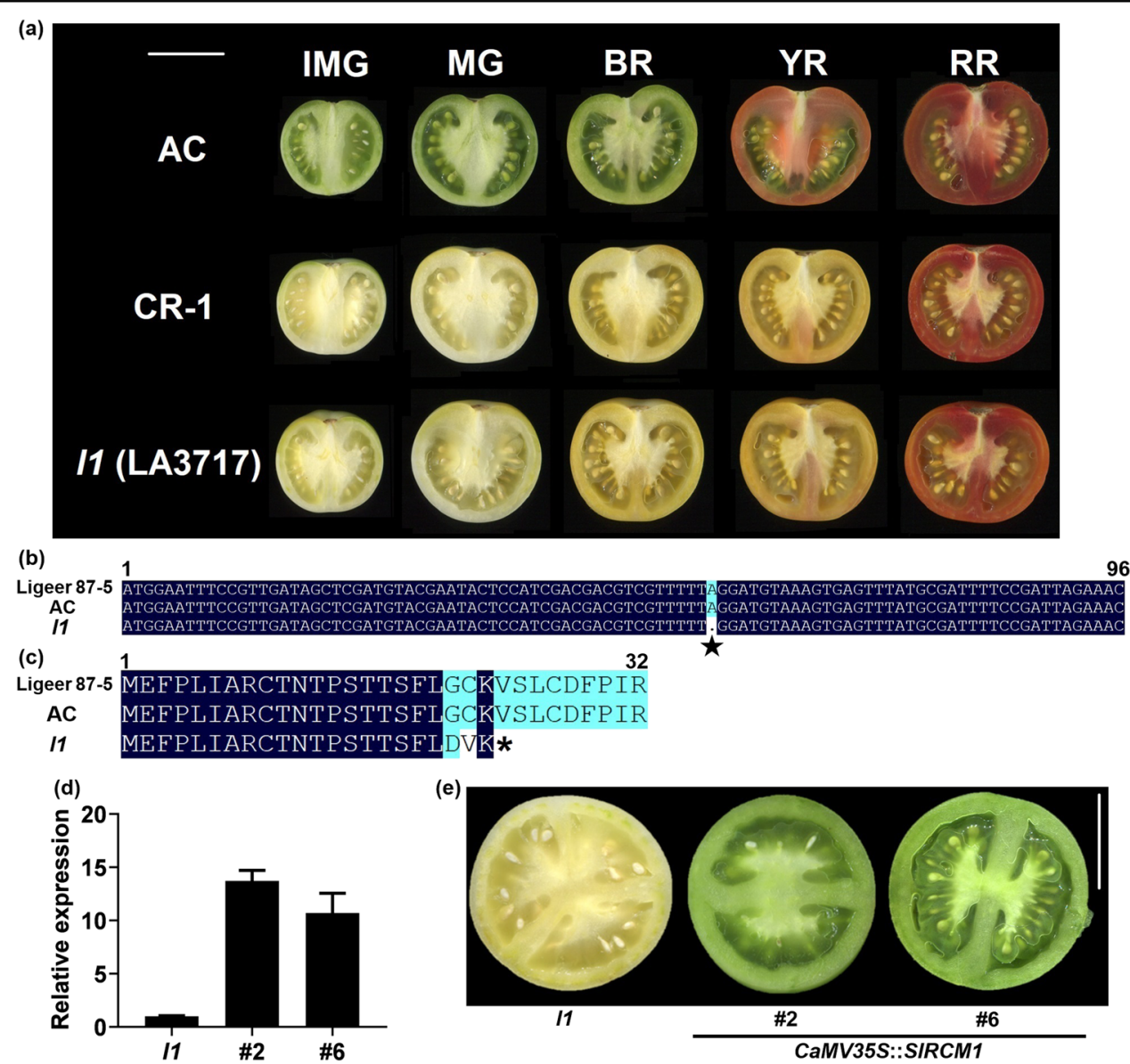

Fig. 5 Lutescent1 is the allele of SIRCM1. a Phenotypes of fruits from the CR-1 line, lutescent1 mutant (11) and AC at different developmental stages. $I M G$, immature green stage; $M G$, mature green stage; $B R$, breaker stage; $Y R$, yellow ripe stage; $R R$, red ripe stage. The $/ 1$ is a mutant in the $A C$ background. Scale bars, $3 \mathrm{~cm}$. b Nucleotide sequence alignment of SIRCM1 fragments in Ligeer 87-5, Ailsa Craig (AC) and 11. The other bases are consistent among Ligeer 87-5, AC and 11. The black star indicates the base at SL2.50ch08_16268 in the first exon of the SIRCM1 gene. c Amino acid sequence alignment of the SIRCM1 protein of Ligeer 87-5, AC and 11. d Relative expression of SIRCM1 in mature green fruits of the SIRCM1overexpressing lines and the control (11). The ACTIN gene (Solyc119005330) was used as an internal control. The data are presented as the means \pm SDs $(n=3)$. e Complementation of the $/ 1$ mutant using SIRCM1. Phenotypes of mature green fruits from /1-and SIRCM1-overexpressing lines were imaged and are shown. Scale bars, $3 \mathrm{~cm}$

in a premature termination mutation in SIRCM1 impaired chloroplast development in the $r c m 1$ mutant. Increasing the chlorophyll content in tomato fruits contributes to improved nutrition ${ }^{36}$. Consistently, the soluble solids content of red ripe fruits of SIRCM1-overexpressing (OE) lines was significantly higher than that of the wild type, whereas the knockout lines (CR) showed the opposite effect (Fig. S7). In addition, the $\mathrm{a}^{*} / \mathrm{b}^{*}$ colorimetric value of the ripe red fruits of the SIRCM1-overexpressing lines (OE) was significantly higher than that of the wild type, while the knockout lines (CR) showed the opposite effect (Fig. S8). Therefore, characterization of the SIRCM1 gene may provide insights into chlorophyll synthesis and chloroplast development in tomato fruits.

The rcm 1 mutant was obtained from an EMSmutagenized population. EMS mutagenesis is widely used in the construction of mutant libraries and in plant functional genomics ${ }^{37,38}$. For instance, the hst1 mutant generated from EMS mutagenesis was crossed with the wild type to map the OsRR22 gene ${ }^{39}$. TILLING analysis ${ }^{40}$ of an EMS-mutagenized population of Arabidopsis showed that EMS mutagenesis produces a large number of single-base substitutions within hotspot segments ${ }^{41}$. In this study, a reduced-chlorophyll mutant, rcm1, was acquired by EMS mutagenesis and crossed with wild type to map the SlRCM1 gene responsible for the yellowishfruit-producing mutant. Due to the close genetic background between the EMS-induced mutant and its wild type, crossing a mutant with its wild type is a fast and effective approach to isolate a gene ${ }^{42,43}$. Two SNPs in the recessive pool with an allele frequency of 1 were found between SL2.50ch08_1 and SL2.50ch08_1010000 (Table 

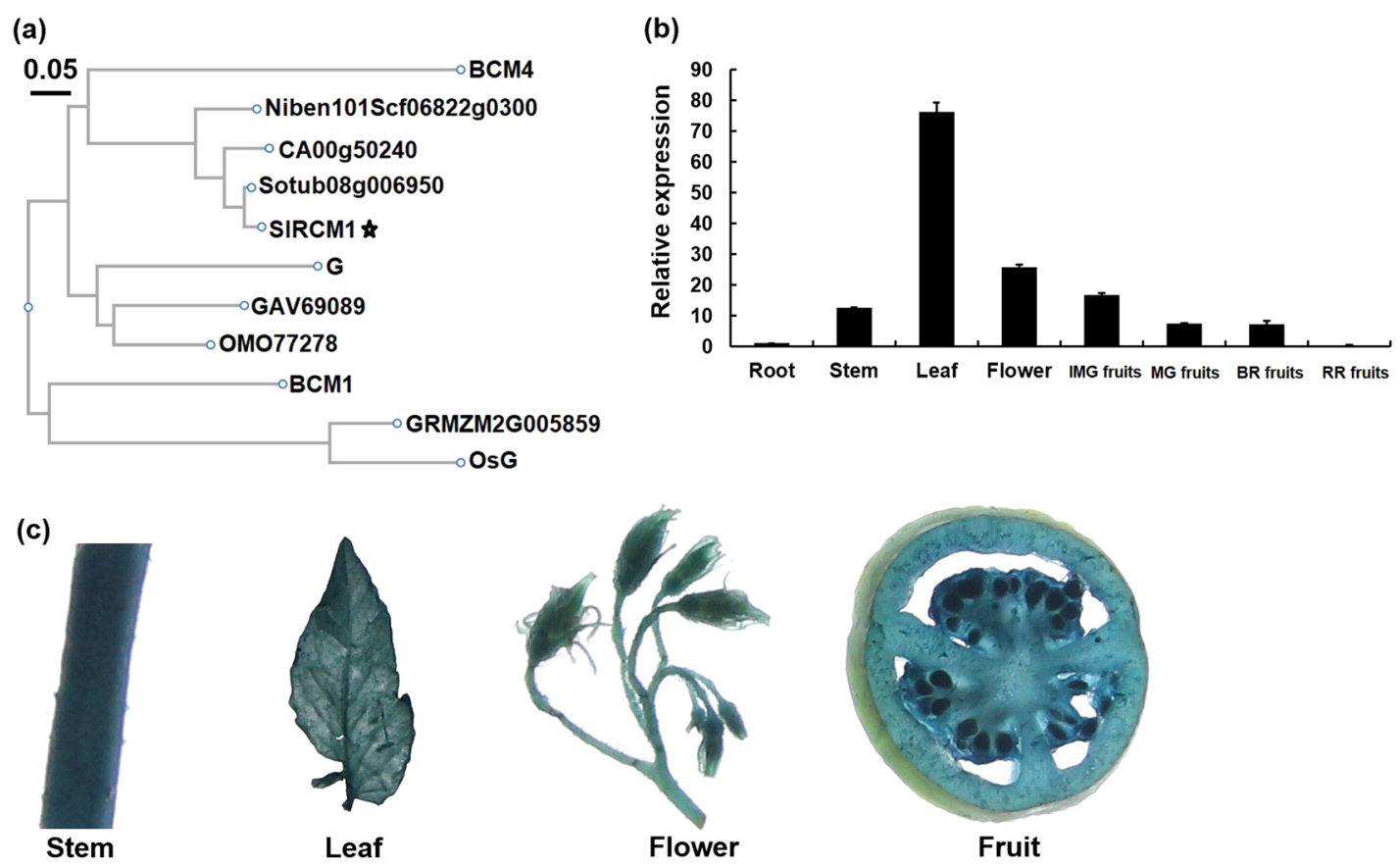

(d)
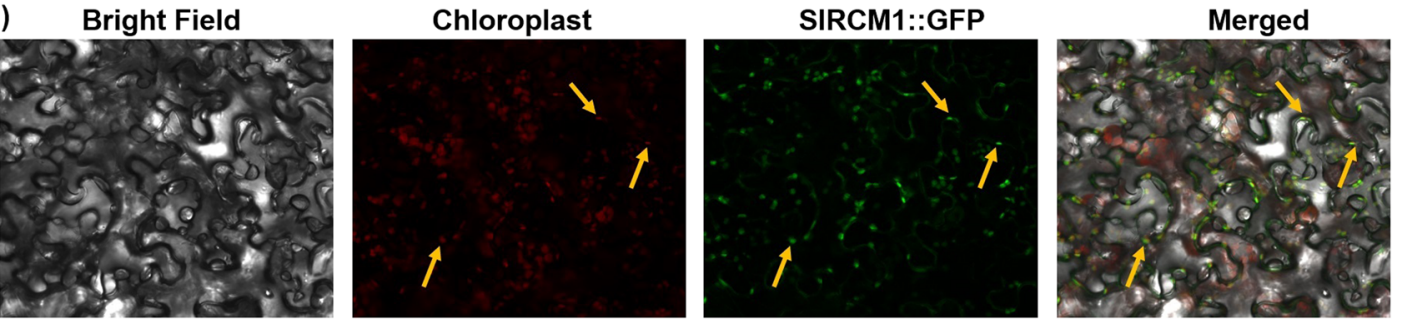

Fig. 6 Characterization of SIRCM1. a Phylogenetic analysis of SIRCM1. The tomato SIRCM1 protein sequence was used to query (via BLAST) the homologous proteins of different species such as soybean (G), potato (Sotub08g006950), pepper (CA00g50240), tobacco (Niben 101Scf06822g03002), Arabidopsis thaliana (BCM1 and BCM4), rice (OsG), maize (GRMZM2G005859), jute (OMO77278), and Cephalotus follicularis (GAV69089) (http://www. ncbi.nlm.nih.gov/). b Relative expression of SIRCM1 in different tissues of Alisa Craig (AC) by qRT-PCR. RNA was extracted from the roots, stems, leaves, flowers, immature green fruits (IMG), mature green fruits (MG), breaker-stage fruits (BR) and red ripe fruits (RR). The ACTIN gene (Solyc11g005330) was used as the internal control. The data are presented as the means \pm SDs $(n=3)$. c The SIRCM1 promoter drives GUS gene expression in AC. Tissues of transgenic tomato plants were stained with X-Gluc. $\mathbf{d}$ Subcellular localization of the SIRCM1 protein. SIRCM1::GFP fusion proteins were transiently expressed in tobacco leaves. Chloroplasts exhibit red fluorescence under a confocal microscope. The arrows highlight the positions of the red and green fluorescence. Scale bars, $25 \mu \mathrm{m}$

S2). Since the SNP at SL2.50ch08_ 394612 was located in an intergenic region, we speculated that it is not a causal SNP for the reduced chlorophyll phenotype of $r \mathrm{~cm} 1$. The SNP $(\mathrm{A} \rightarrow \mathrm{T})$ in the second exon of Solyc08g005010 resulted in a premature stop codon (Fig. 2e). Furthermore, the rcm1 mutant phenotype was rescued by overexpression of the SIRCM1 gene in the $r c m 1$ mutant, which confirmed that SIRCM1 was responsible for the mutant phenotype (Fig. 3). MutMap analysis could therefore be a feasible approach to genetically identify genes in EMSmutagenized mutants.

SIRCM1 has highly conserved functions in both chloroplast development and chlorophyll synthesis. There are two homologous genes of SlRCM1 in Arabidopsis: BCM1 and $B C M 2^{22}$. SIRCM1 was found to share $78 \%$ amino acid sequence identity with BCM1 and $84 \%$ identity with BCM2 (Fig. S1). Previous studies have shown that BCM1 and $B C M 2$ regulate chlorophyll synthesis and chloroplast development in Arabidopsis ${ }^{22}$. Yeast two-hybrid (Y2H) assays, bimolecular fluorescence complementation (BiFC) assays, coimmunoprecipitation (Co-IP) assays and enzyme activity-measuring experiments in Arabidopsis demonstrated that BCM1 interacts with GUN4 to stimulate $\mathrm{Mg}$-chelatase activity and optimize chlorophyll synthesis $^{22}$. BCM1 also interacts with SGR to prevent chlorophyll degradation $^{22}$. SIRCM1's ortholog in soybean 


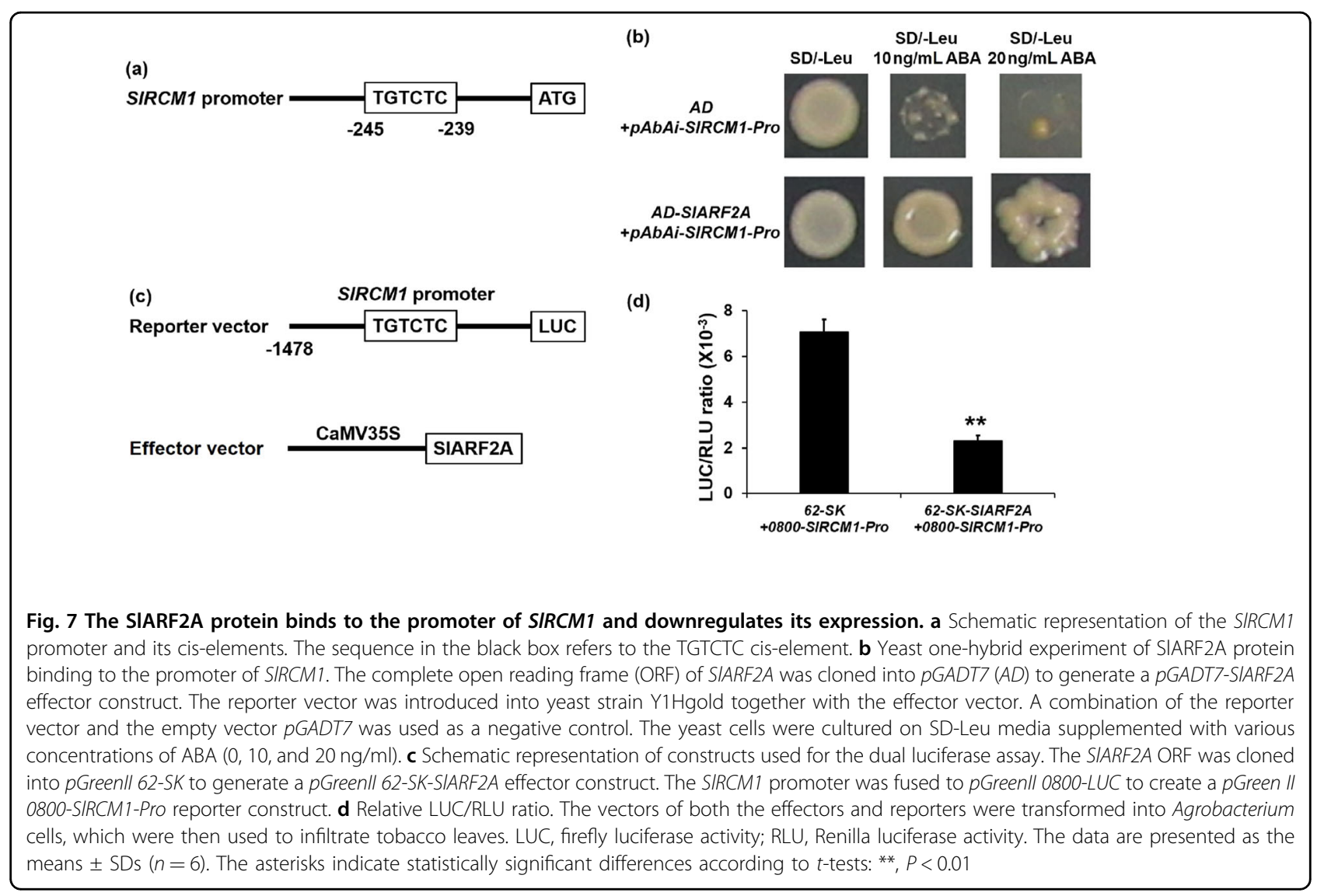

is the stay-green $G$ gene and regulates chlorophyll synthesis and chloroplast development in the seed coat of soybean $^{25}$. However, biological divergence has shown that SIRCM1 regulates chlorophyll synthesis and chloroplast development in fruits, while its ortholog regulates chlorophyll synthesis in the seed coat of soybean ${ }^{25}$ and in the leaves of Arabidopsis ${ }^{22}$.

It has been reported that SIRCM1 orthologs modulate chlorophyll synthesis in the leaves of Arabidopsis and in the seed coat of soybean ${ }^{22,25}$. BCM1 overexpression did not alter the chlorophyll content in Arabidopsis thaliana, but chlorophyll accumulation was inhibited only in the leaves of the $b c m 1$ mutant $^{22}$. In the present study, fruits of the tomato SIRCM1 knockout lines showed a yellowish phenotype due to impaired chloroplasts. The chlorophyll content in the MG-stage fruits of the SIRCM1-overexpressing lines increased significantly. Moreover, chlorophyll was also detected in the RR-stage fruits of the SIRCM1 overexpression lines (Fig. 4). Taken together, these results showed that SIRCM1 has a diversified and strong effect on chlorophyll synthesis and chloroplast development in fruits.

In summary, we have demonstrated that SIRCM1, a chloroplast-targeted ortholog of the BCM1 protein in Arabidopsis and stay-green G protein of soybean, participates in chlorophyll synthesis and chloroplast development in tomato fruits. SlRCM1 modulates the number of thylakoids and the structure of thylakoid membranes in chloroplasts. SIRCM1 was identified as the causal gene at the Lutescent1 locus. An understanding of SIRCM1 provides insights into the molecular mechanism underlying fruit development and target genes for genetic improvement in horticultural crop species.

\section{Materials and methods \\ Plant materials and mutant screening}

A chlorophyll-deficient mutant ( $\mathrm{rcm} 1$ ) was derived from EMS mutagenesis of the processed tomato inbred line Ligeer 87-5. For EMS mutagenesis, Ligeer 87-5 seeds were immersed in 1\% EMS solution and shaken in a shaker for $12 \mathrm{~h}$. The seeds were then rinsed with running water for $10 \mathrm{~min}$ and germinated in an artificial incubator at $30^{\circ} \mathrm{C}$. Mutants were screened in the $\mathrm{M}_{2}$ generation ${ }^{44}$. We constructed an $\mathrm{F}_{2}$ mapping population by crossing Ligeer 875 with $r c m 1$. The $l 1$ mutant (LA3717) in the AC background was obtained from the TGRC (https://tgrc. ucdavis.edu). For the overexpression constructs, SIRCM1 was amplified and cloned into a pHELLSGATE8 vector driven by the CaMV35S promoter and its native promoter ${ }^{45}$. The overexpression vector was introduced into 
Solanum lycopersicum cv. AC and the rcml and $l 1$ mutants through Agrobacterium-mediated transformation. SIRCM1 knockout mutants in the AC background were generated using the CRISPR/Cas9 system $^{46}$. The 2934-bp promoter of the SlRCM1 gene was amplified from $\mathrm{AC}$ and cloned into a $\mathrm{pV} 3 \mathrm{P}$ vector driving $\beta$-glucosidase (GUS), yielding a Pro ${ }^{S l R C M 1}:$ :GUS vector, which was subsequently transformed into AC. The tomato tissues were quickly frozen in liquid nitrogen and stored at $-80{ }^{\circ} \mathrm{C}$. The tomato plants used in this study were grown in a greenhouse, and the primers used in this experiment are listed in Table S4.

\section{Transmission electron microscopy}

The fruits of Ligeer 87-5, the $r \mathrm{~cm} 1$ mutant, $\mathrm{AC}$ and the transgenic lines were isolated and fixed in $0.05 \mathrm{M}$ cacodylate buffer consisting of $2 \%$ glutaraldehyde and dehydrated in ethanol. After embedding in Spurr resin, ultrathin sections of the samples were obtained using a Leica EMUC6 ultramicrotome. A Hitachi H-7650 transmission electron microscope was used to observe the ultrastructure of the plastids.

\section{Determination of the chlorophyll content}

For chlorophyll extraction, fruits of Ligeer 87-5, the rcm 1 mutant, AC and the transgenic lines at different developmental stages were placed in $10 \mathrm{~mL}$ of $80 \%(\mathrm{v} / \mathrm{v})$ acetone in the dark until the tissues became white. Their absorbance was subsequently measured at 646,663 and $470 \mathrm{~nm}$, and the chlorophyll content was measured and calculated as previously reported ${ }^{47}$.

\section{BSA and DNA-seq}

An $\mathrm{F}_{2}$ population comprising 307 individuals was derived from a cross between Ligeer 87-5 and the $\mathrm{rcm} 1$ mutant. In this population, equal amounts of DNA were pooled from 25 plants with a wild-type phenotype and 25 plants with a mutant phenotype. Approximately 25x genome sequences for each pool were generated using the Illumina HiSeq X Ten platform ${ }^{48}$. Due to the close genetic background of the M82 and Ligeer 87-5 processed tomato genotypes, the M82 genome version SL 2.50 (http:// solgenomics.net) was used as the reference genome to facilitate mining of the causal SNPs.

\section{Subcellular localization}

The SlRCM1 coding sequence without the stop codon was amplified from the cDNA of AC and then cloned into a $p H B T$ vector driven by the CaMV35S promoter, yielding CaMV35S::SIRCM1-GFP. This vector was subsequently transformed into Agrobacterium tumefaciens strain GV3101, which was then injected into the leaves of Nicotiana benthamiana ( $N$. benthamiana) as previously described ${ }^{49}$. After $48 \mathrm{~h}$ of incubation at $25^{\circ} \mathrm{C}$, the fluorescence of GFP and RFP in the tobacco leaves was observed using Leica Confocal software. Chloroplasts that exhibit red fluorescence were used as positive controls. The primers used in this experiment are listed in Table S4.

\section{Quantitative reverse transcription PCR}

Total RNA was extracted from frozen tissues using TRIzol reagent (Vazyme, Nanjing, China). RNA was then reverse transcribed into cDNA using a first-strand cDNA Synthesis Kit (Vazyme). The product length ranged from 80 to $200 \mathrm{bp}$. The qRT-PCR was used to determine the transcript levels of genes in 96-well plates with a Roche LightCycler ${ }^{\circledR} 480$ system according to the manufacturer's protocol $^{32}$. The expression of the ACTIN gene (Solyc11g005330) was used as an internal control. The primers used were designed using Primer Premier 5 (Table S4).

\section{Colorimeter-based evaluation of red ripe fruits}

The $a^{*}$ and $b^{*}$ values of ripe red fruits were measured using a CM-5 colorimeter. Three independent points were determined for each fruit assay, and six fruits were measured per line.

\section{Measurement of total soluble solids}

The total soluble solids (Brix) of red ripe fruits were measured using a digital refractometer (PR100, Atago Co., Ltd.). All sample assays were performed for three technical replicates and six biological replicates.

\section{Ethylene assays}

Tomato fruits were collected at 37, 43 and 49 days after flowering. The determination of ethylene content in the tomato fruits was based on previous methods ${ }^{50}$. All the samples consisted of three technical replicates and three biological replicates.

\section{Pollen viability assays}

The pollen of blooming flowers was soaked in I-KI solution ( $1 \% \mathrm{KI}$ and $0.5 \% \mathrm{I}_{2}$ ) for $2 \mathrm{~min}$. The stained pollen was then examined under a low-magnification microscope. The viable pollen was stained dark blue by I-KI.

\section{Chlorophyll fluorescence measurements}

After the leaves of Ligeer 87-5, the $r \mathrm{~cm} 1$ mutant, AC and $L 1-C R$ were conditioned in the dark for $30 \mathrm{~min}$, an imaging pulse amplitude modulated chlorophyll fluorimeter (IMAG-MAXI; Heinz Walz, Effeltrich, Germany) was used to determine chlorophyll fluorescence. The maximal photochemical efficiency of PSII $(\mathrm{Fv} / \mathrm{Fm})$ and the quantum efficiency of PSII (Y(II)) were calculated and determined according to previously reported methods ${ }^{51}$. 


\section{Yeast one-hybrid assays}

The SIARF2A ORF was cloned into a $p G A D T 7$ vector to yield a prey construct. The SIRCM1 promoter was inserted into a $p A b A i$ vector to yield a bait construct. The $p A b A i$ bait vector was then used to transform yeast strain Y1HGold, which was integrated into the yeast genome to generate reporter strains. The prey vector was introduced into the reporter strains and grown for three days on SD/Leu-Ura media. The positive yeast strains were selected and diluted in double-distilled water to an OD600 of 0.1, and $2 \mu \mathrm{L}$ of the suspension was spotted onto $\mathrm{SD} /-\mathrm{Leu}$ media with or without $\mathrm{ABA}(0,10$ and $20 \mathrm{ng} / \mathrm{mL})$, followed by 3 days of incubation at $30{ }^{\circ} \mathrm{C}$. pGADT7 and pAbAi-PSY1-Pro served as negative controls. The primers used in this experiment are listed in Table S4.

\section{Dual luciferase transactivation assays}

To generate an effector construct, the full-length $A R F 2 A$ ORF was inserted into a $p$ GreenII $62-S K$ vector. Similarly, the promoter from SIRCM1 was inserted into $p$ GreenII 0800-LUC to yield a reporter construct. The constructs and the pSoup helper plasmid were simultaneously introduced into Agrobacterium tumefaciens (GV3101). Tobacco leaves were infiltrated with the Agrobacterium strains and harvested three days later. The firefly LUC and Renilla luciferase (RLU) activities were quantified using a dual-luciferase reporter assay system. The transactivation activities were expressed as the ratio of LUC to RLU activity. The primers used in this experiment are listed in Table S4.

\section{Acknowledgements}

We thank Dr. John K. Ahiakpa for his careful reading of the manuscript. This work was supported by grants from the National Key Research and Development Program of China (2018YFD1000800), the National Natural Science Foundation of China (31991182; 31972426), the Wuhan Frontier Projects for Applied Foundation (2019020701011492), the Fundamental Research Funds for the Central Universities (2662018PY073), and the Hubei Provincial Natural Science Foundation of China (2019CFA017).

\section{Author contributions}

Y.Z. and Z.Y. planned and designed the study. G.L., H.Y., L.Y., C.L., J.Y., W.C., Y.W. and P.G. performed the experiments, conducted the fieldwork, and analyzed the data. J.Z. and Y.Z. provided suggestions for experiments. G.L. wrote the manuscript. Y.Z. supervised the project and revised the manuscript.

\section{Conflict of interest}

The authors declare no competing interests.

Supplementary information The online version contains supplementary material available at https://doi.org/10.1038/s41438-021-00563-6.

Received: 4 October 2020 Revised: 24 March 2021 Accepted: 1 April 2021 Published online: 01 June 2021

\section{References}

1. Almeida, J., Perez-Fons, L. \& Fraser, P. D. A transcriptomic, metabolomic and cellular approach to the physiological adaptation of tomato fruit to high temperature. Plant Cell Environ. 13854, 1-9 (2020).
2. Wang, A. Q. et al. The tomato HIGH PIGMENTI/DAMAGED DNA BINDING PROTEIN 1 gene contributes to regulation of fruit ripening. Hortic. Res. 6, 15 (2019).

3. Bruno, A. K. \& Wetzel, C. M. The early light-inducible protein (ELIP) gene is expressed during the chloroplast-to-chromoplast transition in ripening tomato fruit. J. Exp. Bot. 55, 2541-2548 (2004).

4. Powell, A. L. et al. Uniform ripening encodes a Golden 2-like transcription factor regulating tomato fruit chloroplast development. Science 336, $1711-1715$ (2012)

5. Nguyen, C. V. et al. Tomato GOLDEN2-LIKE transcription factors reveal molecular gradients that function during fruit development and ripening. Plant Cell 26, 585-601 (2014).

6. Nadakuduti, S. S., Holdsworth, W. L., Klein, C. L. \& Barry, C. S. KNOX genes influence a gradient of fruit chloroplast development through regulation of GOLDEN2-LIKE expression in tomato. Plant J. 78, 1022-1033 (2014).

7. Pan, Y. et al. Network inference analysis identifies an APRR2-like gene linked to pigment accumulation in tomato and pepper fruits. Plant Physiol. 161, 1476-1485 (2013).

8. Xiong, C. et al. A tomato B-box protein SIBBX20 modulates carotenoid biosynthesis by directly activating PHYTOENE SYNTHASE 1, and is targeted for 265 proteasome-mediated degradation. N. Phytol. 221, 279-294 (2019).

9. Meng, L. et al. BEL1-LIKE HOMEODOMAIN 11 regulates chloroplast development and chlorophyll synthesis in tomato fruit. Plant J. 94, 1126-1140 (2018).

10. Yuan, Y. et al. SIARF10, an auxin response factor, is involved in chlorophyll and sugar accumulation during tomato fruit development. J. Exp. Bot. 69, 5507-5518 (2018).

11. Yuan, Y. et al. Auxin response factor $6 \mathrm{~A}$ regulates photosynthesis, sugar accumulation, and fruit development in tomato. Hortic. Res. 6, 85 (2019).

12. Lim, P. O. et al. Auxin response factor 2 (ARF2) plays a major role in regulating auxin-mediated leaf longevity. J. Exp. Bot. 61, 1419-1430 (2010).

13. Breitel, D. A. et al. AUXIN RESPONSE FACTOR 2 intersects hormonal signals in the regulation of tomato fruit ripening. Plos Genet. 12, e1005903 (2016).

14. Beale, S. I. Green genes gleaned. Trends Plant Sci. 10, 309-312 (2005).

15. Axelsson, E. et al. Recessiveness and dominance in barley mutants deficient in Mg-chelatase subunit D, an AAA protein involved in chlorophyll biosynthesis. Plant Cell 18, 3606-3616 (2006).

16. Alawady, A., Reski, R., Yaronskaya, E. \& Grimm, B. Cloning and expression of the tobacco CHLM sequence encoding Mg protoporphyrin IX methyltransferase and its interaction with Mg chelatase. Plant Mol. Biol. 57, 679-691 (2005).

17. Biswal, A. K. et al. Light intensity-dependent modulation of chlorophyll b biosynthesis and photosynthesis by overexpression of chlorophyllide a oxygenase in tobacco. Plant Physiol. 159, 433-449 (2012).

18. Richter, A. S. et al. Phosphorylation of GENOMES UNCOUPLED 4 alters stimulation of $\mathrm{Mg}$ chelatase activity in angiosperms. Plant Physiol. 172, 1578-1595 (2016).

19. Peter, E. \& Grimm, B. GUN4 is required for posttranslational control of plant tetrapyrrole biosynthesis. Mol. Plant 2, 1198-1210 (2009).

20. Hao, N. et al. CsMYB36 is involved in the formation of yellow green peel in cucumber (Cucumis sativus L.). Theor. Appl. Genet. 131, 1659-1669 (2018).

21. Wang, N. et al. Defect in Brnym 1, a magnesium-dechelatase protein, causes a stay-green phenotype in an EMS-mutagenized Chinese cabbage (Brassica campestris L. ssp. pekinensis) line. Hortic. Res. 7, 8 (2020).

22. Wang, P., Richter, A. S., Kleeberg, J. R. W., Geimer, S. \& Grimm, B. Posttranslational coordination of chlorophyll biosynthesis and breakdown by BCMs maintains chlorophyll homeostasis during leaf development. Nat. Commun. 11, 1254 (2020).

23. Czarnecki, O. et al. An Arabidopsis GluTR binding protein mediates spatial separation of 5-aminolevulinic acid synthesis in chloroplasts. Plant Cell 23, 4476-4491 (2011).

24. Pontier, D., Albrieux, C., Joyard, J., Lagrange, T. \& Block, M. A. Knock-out of the magnesium protoporphyrin IX methyltransferase gene in Arabidopsis effects on chloroplast development and on chloroplast-to-nucleus signaling. J. Biol. Chem. 282, 2297-2304 (2007).

25. Wang, $\mathrm{M}$. et al. Parallel selection on a dormancy gene during domestication of crops from multiple families. Nat. Genet. 50, 1435-1441 (2018).

26. Barry, C. S. et al. Altered chloroplast development and delayed fruit ripening caused by mutations in a zinc metalloprotease at the lutescent2 locus of tomato. Plant Physiol. 159, 1086-1098 (2012).

27. Almeida, J. et al. Fruits from ripening impaired, chlorophyll degraded and jasmonate insensitive tomato mutants have altered tocopherol content and composition. Phytochemistry 111, 72-83 (2015). 
28. Mizzotti, C. et al. Time-course transcriptome analysis of arabidopsis siliques discloses genes essential for fruit development and maturation. Plant Physiol. 178, 1249-1268 (2018).

29. Menda, N., Semel, Y., Peled, D., Eshed, Y. \& Zamir, D. In silico screening of a saturated mutation library of tomato. Plant J. 38 (2004).

30. Saito, T. et al. TOMATOMA: a novel tomato mutant database distributing Micro-Tom mutant collections. Plant Cell Physiol. 52, 283-296 (2011).

31. Tanksley, S. D. et al. High density molecular linkage maps of the tomato and potato genomes. Genetics 132, 1141-1160 (1992).

32. Xie, Q. et al. The HD-Zip IV transcription factor SIHDZIV8 controls multicellular trichome morphology by regulating the expression of Hairless-2. J. Exp. Bot. (2020).

33. Bobik, K. et al. The essential chloroplast ribosomal protein UL15c interacts with the chloroplast RNA helicase ISE2 and affects intercellular trafficking through plasmodesmata. N. Phytol. 221, 850-865 (2019).

34. Yuan, Y. J. et al. SIARF10, an auxin response factor, is involved in chlorophyll and sugar accumulation during tomato fruit development. J. Exp. Bot. 69, 5507-5518 (2018).

35. Croce, R. \& van Amerongen, $H$. Natural strategies for photosynthetic light harvesting. Nat. Chem. Biol. 10, 492-501 (2014).

36. Cocaliadis, M. F., Fernandez-Munoz, R., Pons, C., Orzaez, D. \& Granell, A. Increasing tomato fruit quality by enhancing fruit chloroplast function. A double-edged sword? Exp. Bot. 65, 4589-4598 (2014).

37. Henry, I. M. et al. Efficient genome-wide detection and cataloging of EMSinduced mutations using exome capture and next-generation sequencing. Plant Cell 26, 1382-1397 (2014)

38. Sashidhar, N., Harloff, H. J. \& Jung, C. Identification of phytic acid mutants in oilseed rape (Brassica napus) by large-scale screening of mutant populations through amplicon sequencing. N. Phytol. 225, 2022-2034 (2020).

39. Takagi, $H$. et al. MutMap accelerates breeding of a salt-tolerant rice cultivar. Nat. Biotechnol. 33, 445-449 (2015).
40. Lakhssassi, N. et al. Soybean TILLING-by-Sequencing reveals the role of nove GmSACPD members in the unsaturated fatty acid biosynthesis while maintaining healthy nodules. J. Exp. Bot. 71, 6969-6987 (2020).

41. Greene, E. A. et al. Spectrum of chemically induced mutations from a large-scale reverse-genetic screen in Arabidopsis. Genetics 164, 731-740 (2003).

42. Abe, A. et al. Genome sequencing reveals agronomically important loci in rice using MutMap. Nat. Biotechnol. 30, 174-178 (2012).

43. Fekih, R. et al. MutMap plus: genetic mapping and mutant identification without crossing in rice. PLOS ONE 8, e68529 (2013).

44. Saito, T. et al. TOMATOMA: a novel tomato mutant database distributing micro-tom mutant collections. Plant Cell Physiol. 52, 283-296 (2011).

45. Chang, J. et al. Hair, encoding a single $\mathrm{C} 2 \mathrm{H} 2$ zinc-finger protein, regulates multicellular trichome formation in tomato. Plant J. 96, 90-102 (2018).

46. Cui, L. et al. miR156a-targeted SBP-Box transcription factor SISPL13 regulates inflorescence morphogenesis by directly activating SFT in tomato. Plant Biotechnol. J. 18, 1670-1682 (2020).

47. Lichtenthaler, H. K. Chlorophylls and carotenoids-pigments of photosynthetic biomembranes. Method. Enzymol. 148, 350-382 (1987)

48. Meynert, A. M., Ansari, M., FitzPatrick, D. R. \& Taylor, M. S. Variant detection sensitivity and biases in whole genome and exome sequencing. Bmc Bioinformatics 15, 247 (2014).

49. Tian, Z . et al. The potato ERF transcription factor StERF3 negatively regulates resistance to phytophthora infestans and salt tolerance in potato. Plant Cell Physiol. 56, 992-1005 (2015).

50. Vrebalov, J. et al. A MADS-box gene necessary for fruit ripening at the tomato ripening-inhibitor (Rin) locus. Science 296, 343-346 (2002).

51. Li, H. et al. Hydrogen peroxide mediates abscisic acid-induced HSP70 accumulation and heat tolerance in grafted cucumber plants. Plant Cell Environ. 37, 2768-2780 (2014). 